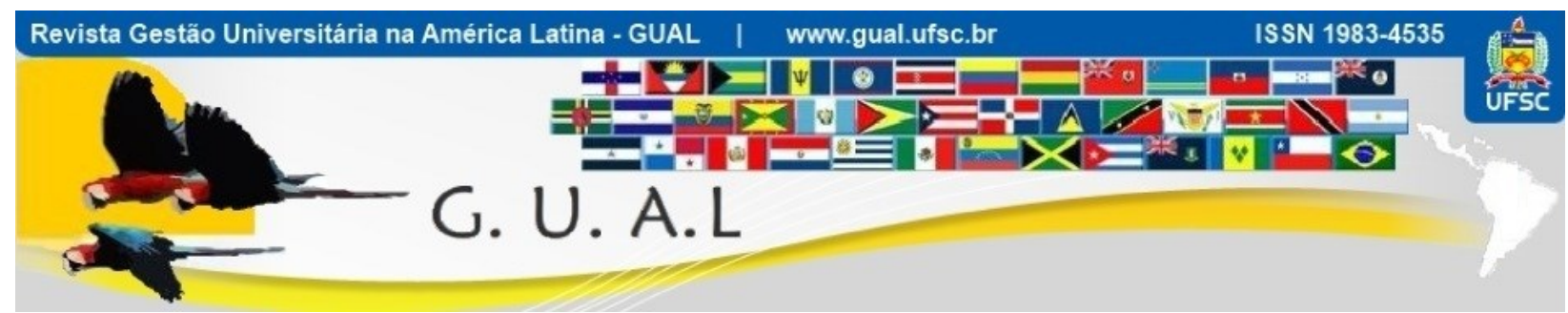

DOI: http://dx.doi.org/10.5007/1983-4535.2015v8n4p101

\title{
CALIDAD DE LA EDUCACIÓN SUPERIOR: PERCEPCIONES DE LOS ESTUDIANTES UNIVERSITARIOS ARGENTINOS
}

\author{
QUALITY OF HIGHER EDUCATION: PERCEPTIONS OF ARGENTINIAN \\ UNIVERSITY STUDENTS
}

María Paz Andreasen, Mestre Universidad Nacional de La Plata paz.andreasen@econo.unlp.edu.ar

María de la Paz Colombo, Graduada Universidad Nacional de La Plata paz.colombo@econo.unlp.edu.ar

Gabriela Fernanda Mollo Brisco, Mestre Universidad Nacional de La Plata gabriela.mollo@econo.unlp.edu.ar

Juan José Gilli, Doutor Universidad Nacional de La Plata juanjose.gilli@econo.unlp.edu.ar

Martín López Armengol, Mestre Universidad Nacional de La Plata martin.lopezarmengol@econo.unlp.edu.ar

Recebido em 05/dezembro/2014

Aprovado em 05/abril/2015

Sistema de Avaliação: Double Blind Review

Esta obra está sob uma Licença Creative Commons Atribuição-Uso. 


\title{
CALIDAD DE LA EDUCACIÓN SUPERIOR: PERCEPCIONES DE LOS ESTUDIANTES \\ UNIVERSITARIOS ARGENTINOS \\ DOI: http://dx.doi.org/10.5007/1983-4535.2015v8n4p101
}

\section{RESUMEN}

La universidad pública es un agente educativo, social y económico de primer orden en la sociedad del conocimiento. Las universidades son probablemente las instituciones que mayor impacto y valor añadido aportan a su territorio, ya sea por la vía de la formación y el capital humano, la investigación científica, el desarrollo social y cultural o la innovación y el progreso económico. Bajo estas premisas es que las universidades se posicionan como pilares fundamentales donde se sustenta el nuevo modelo de sociedad y, por ello, asegurar la calidad de la educación superior es, o debería ser, una temática ineludible en la agenda de todos los sectores involucrados en la gestión universitaria. Sin embargo, aún hoy existen dificultades para arribar a una conceptualización del término "calidad" que incluya la opinión de todos los estamentos involucrados en el proceso educativo y para lograr consenso sobre cuáles son los determinantes que más influyen sobre la misma en el ámbito universitario. Ante esta realidad, y cerrando una serie de investigaciones en el marco de la RED ECUALE , este artículo pretende ser un aporte empírico para el estudio de la calidad educativa universitaria indagando acerca de las percepciones que priman entre los/as estudiantes de la Universidad Nacional de La Plata sobre la definición de calidad y los factores que mayor impacto poseen sobre ella. Entre los resultados más importantes ha surgido que los estudiantes asocian el concepto de calidad con la capacidad de la educación superior de generarles un espíritu crítico, de dotarlos de capacidades para el aprendizaje autónomo y de hacerlos asumir un fuerte compromiso social. En cuanto a sus factores determinantes, se ha evidenciado que para los estudiantes las cuestiones más relevantes que afectan a la calidad se encuentran asociadas a sus propias características y condiciones por sobre los aspectos asociados al profesorado y a las cuestiones institucionales.

Palabras clave: Calidad enseñanza superior. Gestión educativa. Impacto social universitario. Estudiantes universitarios.

\begin{abstract}
The public university is an educational, social and economic agent of first order in the knowledge society. Universities are the institutions which probably provide greater impact and added value to its territory, whether by formation mode and human capital, scientific research, social and cultural development or innovation and economic progress. Under these assumptions is that universities have become essential pillars where the new model of society is based and, therefore, guarantee the quality of higher education is, or should be, an unavoidable topic on the agenda of all sectors involved in university management. However, there are still difficulties to arrive at a definition of the term "quality" to include the views of all sectors involved in the educational process and to reach consensus on the determinants that produce the greatest influence on quality in the university environment. Given this reality, and closing a series of investigations under the ECUALE RED1, this article is intended as an empirical contribution to the study of university education quality inquiring about perceptions that prevail between the National University of La Plata students on the definition of quality and the factors that have most impact on it. Among the most important findings have emerged that students associate the concept of quality with the ability of higher education to generate on them a critical spirit, to equip them with skills for independent learning and make them assume a strong social commitment. Regarding its determinants, it has been demonstrated that for students the most relevant issues affecting quality are associated with their own characteristics and conditions over the issues associated with the professors and institutional issues.
\end{abstract}

Keywords: Quality Higher Education. Educational Management. Academic Social Impact. University Students. 


\section{INTRODUCCIÓN}

Dada la relevancia de la calidad educativa, los estudios y propuestas doctrinales sobre la calidad en el ámbito de la enseñanza superior, han proliferado desde la década del noventa en nuestro país y en América Latina.

En este marco es necesario reconocer que el terreno de la educación universitaria presenta particularidades que impiden que, los conceptos que ya han tenido éxito en las actividades industriales, sean extrapolados acríticamente. En tal sentido, el principal obstáculo que se presenta es que existen dificultades para arribar a una conceptualización del término "calidad" que satisfaga las expectativas de los diferentes actores involucrados en el proceso educativo.

El objetivo de este trabajo es realizar un aporte empírico para el estudio de la calidad educativa universitaria indagando sobre las opiniones de los estudiantes de la Universidad Nacional de La Plata acerca del significado que tiene, para ellos y para las instituciones en las que estudian, el concepto de "calidad de la educación superior". Asimismo se pretende lograr una primera aproximación sobre cuáles son los factores que estos consideran que actúan favorablemente sobre dicha calidad en el contexto de la enseñanza universitaria argentina.

Específicamente, en términos metodológicos, para encarar el estudio se ha trabajado a partir de técnicas cuantitativas de recolección de datos, más precisamente, se ha recurrido a un cuestionario cerrado autoadministrado, diseñado con un formato Likert.

\section{MARCO CONCEPTUAL}

El siglo XXI ha visto surgir un nuevo paradigma de sociedad, un modelo donde la información entendida como conocimiento aparece como el cimiento del desarrollo económico, político y social de esta nueva era. Peter Drucker (1993) es reconocido como uno de los primeros en percibir la transformación de una sociedad industrial manufacturera a una de servicios, donde el conocimiento se convierte en el recurso básico. Drucker acuñó términos básicos como trabajo de conocimiento o trabajador del conocimiento. Es preciso recalcar la repercusión y el impacto que la actividad de las universidades (especialmente las públicas) genera en la sociedad y en la economía de una región. Bajo estas premisas, es que las Universidades se posicionan como pilares fundamentales donde se sustenta el nuevo modelo de sociedad; y asegurar la calidad de la educación superior se transforma en un tema de especial trascendencia. 


\section{CALIDAD DE LA EDUCACIÓN SUPERIOR: PERCEPCIONES DE LOS ESTUDIANTES UNIVERSITARIOS ARGENTINOS \\ DOI: http://dx.doi.org/10.5007/1983-4535.2015v8n4p101}

En este marco es necesario reconocer que el terreno de la enseñanza superior presenta particularidades que impiden que los conceptos que ya han tenido éxito en las actividades industriales, sean extrapolados acríticamente. En tal sentido, el principal obstáculo que se presenta es que, aún hoy, existen dificultades para arribar a una conceptualización del término "calidad" que incluya la opinión de todos los estamentos involucrados en el proceso educativo.

La exposición más completa y aceptada de los significados que puede adoptar la calidad en el contexto universitario es la de Harvey y Green (1993). A continuación se exponen de manera resumida los conceptos de calidad desarrollados por estos autores y sus principales características.

\section{CONCEPCIÓN DE CALIDAD Y SUS CARACTERISTICAS}

\section{La calidad como excepcional}

Visión tradicional de la calidad: basada en la distinción y la exclusividad; las universidades personalizan la calidad.

Visión de la calidad ligada a la excelencia: Superación de unos estándares muy altos. Para conseguir la excelencia en los resultados se requiere lo mejor: mejores profesores, mejores alumnos, mejores infraestructuras, etc. Este enfoque no da importancia al proceso.

Visión de la calidad consistente en el cumplimiento de unos estándares mínimos. La calidad según este enfoque es atribuida a todos los que cumplen esos estándares. Supone implícitamente que los estándares son objetivos

\section{La calidad como perfección o consistencia}

Se relaciona con la idea de cero defectos, con hacer las cosas bien la primera vez. Supone intrínsecamente la existencia de una cultura de la calidad. La universidad se compone de nódulos y es responsabilidad de cada nódulo que su output cumpla los requisitos del input del proceso siguiente.

\section{La calidad como adecuación a una finalidad}

La calidad se concibe como el ajuste a un propósito. En este enfoque la calidad se juzga por el output, no por el proceso. Se define la calidad en términos de satisfacer los requisitos, necesidades o deseos del cliente. Relaciona la calidad con la funcionalidad.

La calidad es definida en términos de que la institución cumpla los objetivos que ha establecido, es decir, su misión.

\section{La calidad como valor por dinero}

La calidad se relaciona con la eficiencia económica, con el rendimiento de una inversión. En el centro de este enfoque está la idea de rendición de cuentas o accountability.

\section{La calidad como transformación}

La calidad radica en desarrollar las capacidades del estudiante y en capacitarle para su propia transformación. Este enfoque va más allá de los cambios que pueden operar a nivel académico e incorpora nociones asociadas a la ética y al compromiso social.

Cuadro 1 Concepciones de Calidad de la Educación Superior y sus características Fuente: Adaptado de Barrenetxea, 2005 
Las cinco nociones de calidad propuestas por Harvey y Green (1993) pueden ser ordenadas en el plano generado por dos dimensiones (Gráfico 1). El eje horizontal representa el grado en que cada noción de calidad se deriva de un referente interno a la institución o, de algún modo, externo (ajeno) a él. El eje vertical propone una división diferente de los conceptos de calidad. La parte inferior de dicho eje reúne las definiciones tradicionales sobre gestión de la calidad (tanto en el ámbito específico de las instituciones universitarias como en el más general de la gestión empresarial), mientras que, en la parte superior de este eje se refleja una visión más moderna del fenómeno.

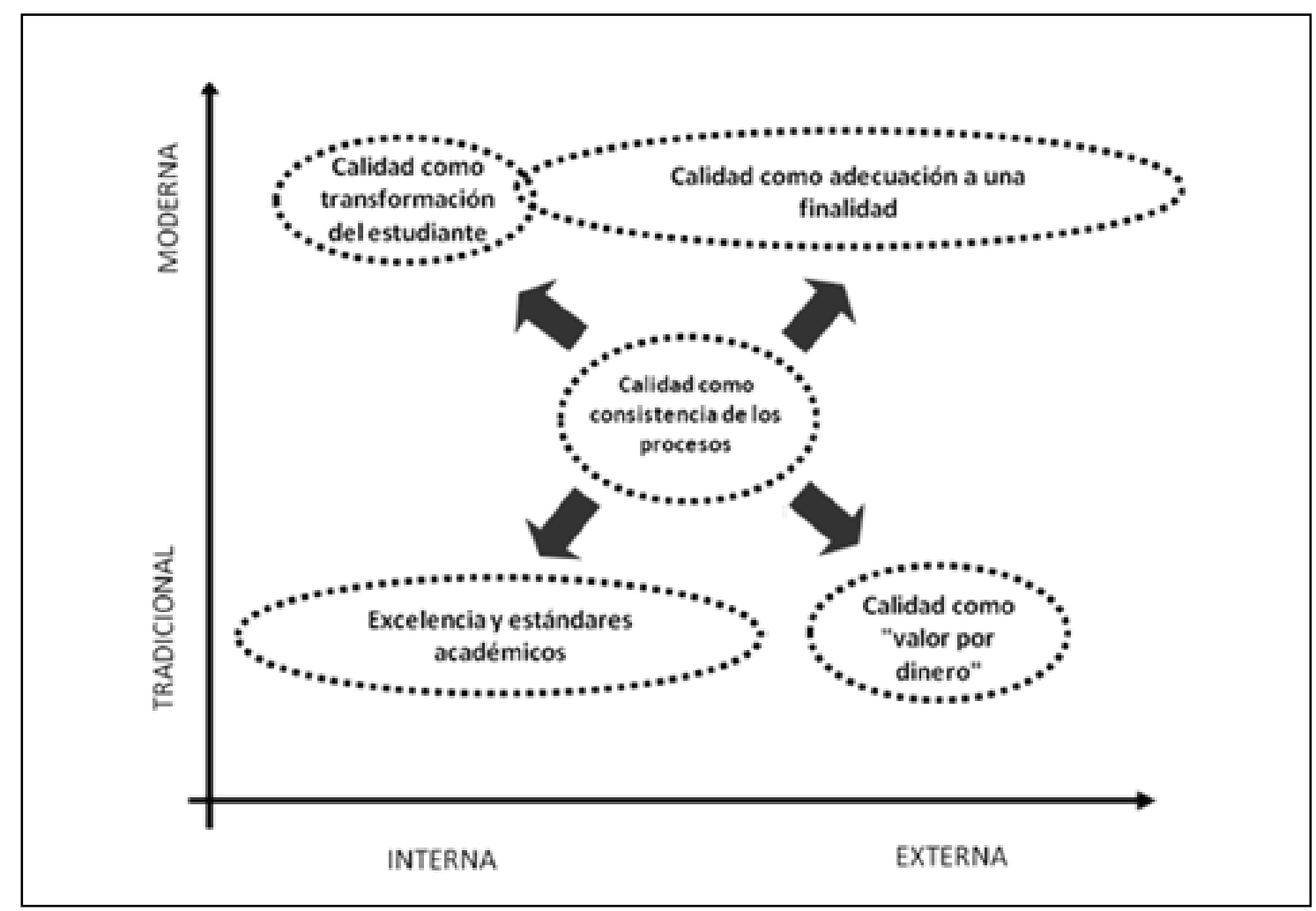

Gráfico 1 Los conceptos de calidad (Harvey y Green, 1993) en un espacio bidimensional Fuente: López Armengol, M. A. y Colombo, M. P. (coords.), 2011

Naturalmente, estas cinco nociones de calidad no son del todo incompatibles, y menos aun cuando lo que se trata de reconocer es su presencia en el universo simbólico de los actores (en este caso los que actúan en las instituciones universitarias). Es decir, no debe sorprender que un mismo individuo defienda varios modos de entender la calidad.

Ahora bien, profundizando aún más sobre esta temática, resulta importante analizar de una forma más concreta y menos conceptual, cuáles son los factores que influyen directamente sobre la calidad ya que serán estos últimos sobre los que luego se podrá actuar en forma efectiva a nivel institucional. 
Son numerosos los autores que han abordado esta temática desde distintas perspectivas. Martínez M. C y Romero M. A. en su trabajo titulado "Calidad de la educación e integración de las nuevas tecnologías" (1999) mencionan siete factores que inciden en la calidad de la enseñanza:

1) La adquisición e implementación de tecnologías avanzadas de información y comunicación.

2) La orientación de las metas educativas hacia a la esfera personal como a la social y la contribución al desarrollo económico y sociocultural de la comunidad.

3) El fomento de los aspectos referidos a la colaboración, ya sea con grupos externos al ámbito educativo, coordinación con otras instituciones educativas no universitarias, colegialidad entre profesores, departamentos, alumnos e instituciones del sector universitario.

4) La optimización del ratio alumnos/aulas.

5) La mejora de la motivación del profesorado.

6) El fomento de la actividad investigadora.

7) La elaboración e implementación de un plan de evaluación (de la calidad en la enseñanza por parte de los estudiantes, de la relación entre institución y estudiantes, de la valoración de la universidad como entorno de trabajo, de las relaciones de la universidad con otras instituciones, de los logros por parte de los estudiantes, etc).

Por su parte Mora Ruiz (1991) ha efectuado una categorización de los factores que estima afectan mayormente a la calidad de las universidades en cuatro bloques: uno asociado a las características de los alumnos, otro que incluye los índices referentes al funcionamiento del proceso, un tercero que se relaciona con las instituciones y un cuarto grupo que contiene los factores asociados a los resultados del proceso.

Analizando el tema en función de la incidencia o importancia relativa de estos factores, Torres González (1996) afirma que la educación mejorará en la medida en que lo haga el cuerpo docente y Gento Palacios (1995) proclama que lo fundamental es que el docente promueva procesos de autoaprendizaje en los alumnos, un clima apropiado favorable a la comunicación, un fácil acceso a las fuentes de información y a la adquisición de conocimientos, a la vez que seleccione experiencias formativas que conduzcan al desarrollo intelectual de los estudiantes. En esta misma línea, Ruiz Carrascosa (1996) señala que la clave para la calidad está dada por la necesidad de que el profesorado investigue en el desarrollo de su práctica docente, de modo que responda a los problemas que puedan presentarse en los centros y en las aulas.

\section{OBJETIVOS}


El objetivo de este trabajo es, fundamentalmente, describir las opiniones de los estudiantes de la Universidad Nacional de La Plata acerca del significado que tiene, para ellos y para las instituciones en las que estudian, el concepto de "calidad de la enseñanza universitaria". En segunda instancia, se espera brindar una primera aproximación sobre cuáles son los factores que estos consideran que actúan favorablemente sobre dicha calidad en el contexto de la educación superior argentina.

\section{METODOLOGÍA}

En el presente trabajo se exponen los resultados de la última investigación realizada en el marco de la Red ECUALE: los del Proyecto CESPEA (La Calidad en la Educación Superior: un estudio sobre la Percepción de los Estudiantes de Argentina), el cual ha tenido como unidad de análisis a los estudiantes de la Universidad Nacional de La Plata. La población sobre la cual se realizó el muestreo fue 102.733 estudiantes de 17 Unidades Académicas de la Universidad Nacional de La Plata. Con estos datos se realizó un muestreo estratificado para una población infinita y se definió una muestra de 573 casos.

En cuanto a la estrategia de relevamiento se optó por la utilización de un cuestionario cerrado autoadministrado de manera presencial, pues resultaba más sencillo ubicar los estudiantes físicamente en las aulas.

Para la confección de la encuesta se recurrió al formato Likert. El cuestionario empleado se encontraba conformado por tres grandes bloques:

El primero orientado al análisis de la concepción de calidad. Para tal fin se indagó sobre el grado de identificación de los encuestados con diversas concepciones de calidad. Para esto se recurrió a la operativización de los conceptos planteados por Harvey y Green y desarrollados oportunamente. Específicamente se utilizaron las siguientes concepciones de calidad:

- La calidad consiste en desarrollar las capacidades del estudiante para avanzar en su propia transformación (gestionar sus propios conocimientos). (C1)

- La calidad consiste en formar estudiantes capaces de asumir un compromiso social. (C2)

- La calidad consiste en el cumplimiento de los objetivos y la misión que ha establecido la institución. (C3)

- La calidad consiste en satisfacer las expectativas de todos los involucrados (estudiantes, profesores, instituciones, sociedad). (C4) 
- La calidad consiste en satisfacer las expectativas de los estudiantes y de las organizaciones donde trabajarán los egresados. (C5)

- La calidad consiste en el cumplimiento de objetivos y de estándares académicos y científicos establecidos. (C6)

- La calidad consiste en conseguir la eficiencia: obtener los mejores resultados con el menor uso de recursos. (C7)

En el Gráfico 2 se presenta esquemáticamente la relación que se ha establecido entre las variables operativas antes definidas y los conceptos originales que se han oportunamente expuestos en el Gráfico 1.

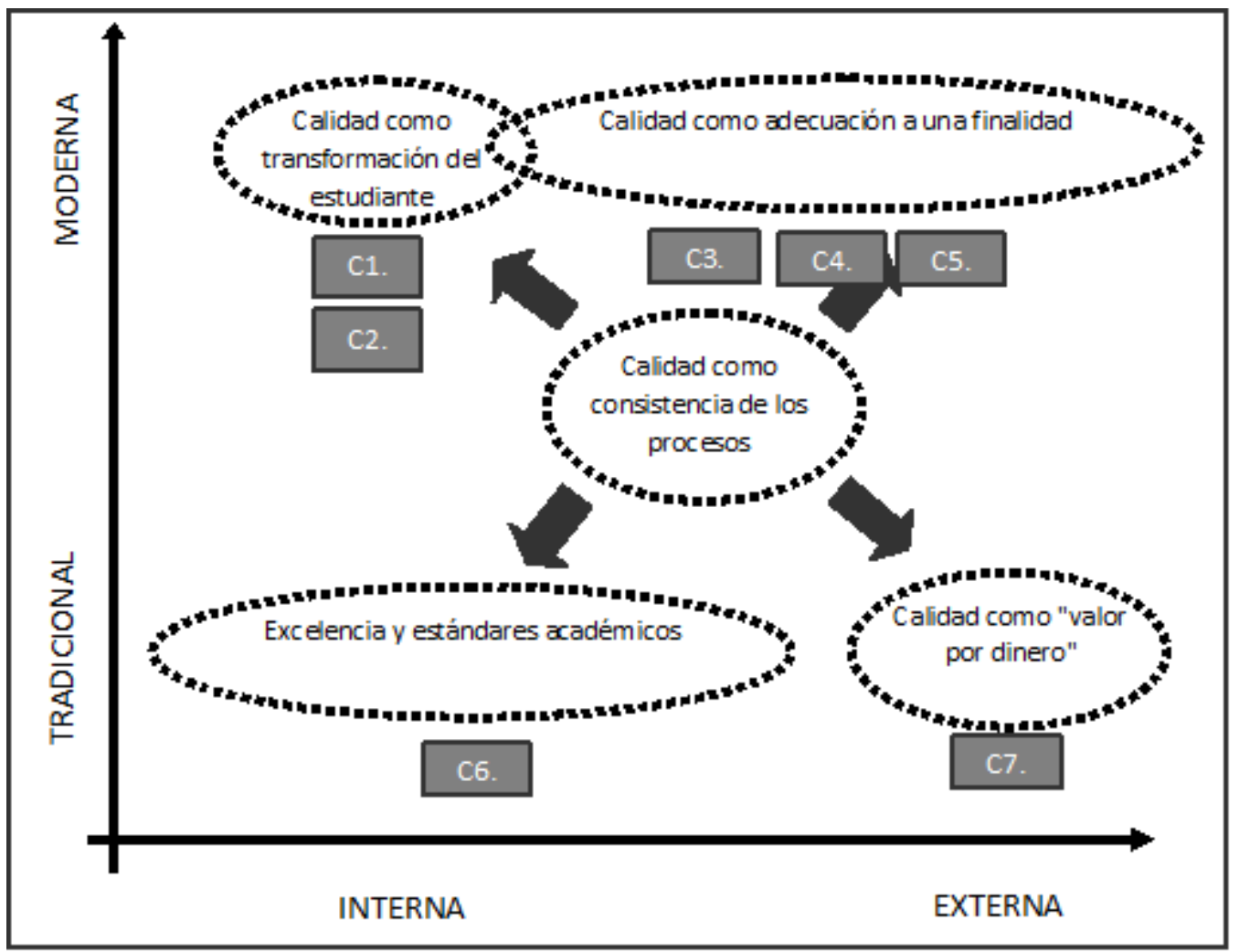

Gráfico 2 Los conceptos de calidad (Harvey y Green, 1993) y los ítems diseñados para operacionalizarlos. Fuente: López Armengol, M. A. y Colombo, M. P. (coords.), 2011

El segundo de los bloques del cuestionario se encuentra orientado al análisis de los factores que afectan la mejora de la calidad de la educación universitaria. Este bloque se compone de dos interrogantes: el primero indaga sobre la opinión de los encuestados respecto al grado de importancia que los factores indicados poseen en la mejora de la educación universitaria $y$, el segundo, les consulta sobre el grado en que perciben que los diferentes factores se encuentran presentes en su universidad.

Estos factores se diferenciaron en tres grupos para su análisis, aquellos vinculados al Profesorado; los vinculados a la Institución Universitaria y aquellos relacionados con los 
Estudiantes. Sin embargo, los mismos se preguntaron en forma conjunta e indistintamente en las encuestas.

Por último, el tercer bloque de los cuestionarios, englobaba lo que se conoce habitualmente como variables de control. Donde se consultó sobre el área de conocimiento al cual pertenece la Facultad a la que pertenecía el estudiante, la carrera por la que había optado, su género, edad y grado de avance en la carrera que se encontraba cursando.

\section{RESULTADOS}

A continuación se expone un análisis descriptivo de los principales resultados obtenidos en el estudio. Debe tenerse en cuenta que se trata de un análisis no condicionado, con lo cual no pueden extraerse conclusiones acerca de la incidencia de determinadas características sobre los resultados, y mucho menos sobre su causalidad. Se trata simplemente de una descripción de las frecuencias relativas (altas y muy altas) -FRA- de las respuestas obtenidas.

\subsection{GRADO DE ADHESIÓN DE LOS ESTUDIANTES DE LA UNIVERSIDAD NACIONAL DE LA PLATA A DIFERENTES CONCEPCIONES DE CALIDAD}

Tal como se ha mencionado anteriormente, el estudio realizado indaga sobre la opinión de los estudiantes universitarios acerca de siete modos alternativos de definir el concepto de calidad. En una primera instancia se les solicitó su identificación personal con cada una de las definiciones y, posteriormente, cuál consideraban que era la identificación de su facultad con los mismos conceptos. Los resultados expresados por los estudiantes se presentan en el Cuadro 2 donde se evidencia una marcada diferencia a favor de las concepciones de calidad que se podrían inscribir dentro de las definiciones más modernas y asociadas a los agentes "internos" de la institución. Es decir que los mencionados estudiantes adhieren a los conceptos vinculados con el poder transformador de la enseñanza universitaria. 


\section{CALIDAD DE LA EDUCACIÓN SUPERIOR: PERCEPCIONES DE LOS ESTUDIANTES \\ UNIVERSITARIOS ARGENTINOS \\ DOI: http://dx.doi.org/10.5007/1983-4535.2015v8n4p101}

\section{CONCEPTO DE CALIDAD}

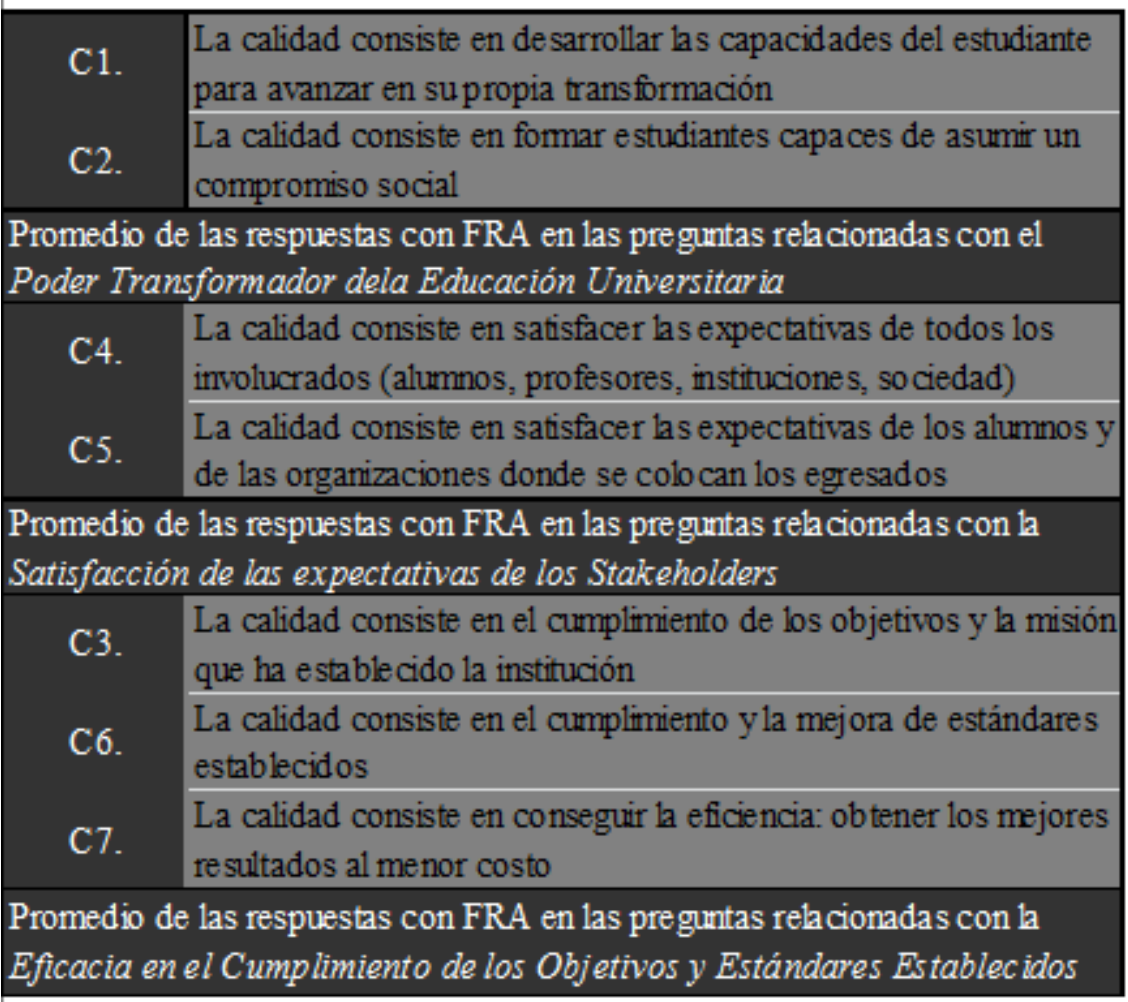

\begin{tabular}{|c|}
\hline $\begin{array}{c}\text { ESTUDIAN } \\
\text { TES }\end{array}$ \\
\hline $84 \%$ \\
$75 \%$ \\
\hline $\mathbf{8 0} \%$ \\
\hline $65 \%$ \\
$53 \%$ \\
\hline $\mathbf{5 9} \%$ \\
\hline $51 \%$ \\
\hline $57 \%$ \\
\hline $42 \%$ \\
\hline $\mathbf{5 0} \%$ \\
\hline
\end{tabular}

Cuadro 2 Grado de adhesión de los estudiantes a diferentes conceptos de calidad (FRA) Fuente: Elaboración Propia

En función a los resultados obtenidos y tal como se mencionó anteriormente, los estudiantes universitarios parecerían defender principalmente definiciones asociadas a conceptualizaciones más modernas de calidad las cuales se encuentran relacionadas al poder transformador de la educación universitaria (la necesidad de formar estudiantes con capacidades para avanzar en su propia transformación y para asumir un compromiso social).

Los encuestados consideran indiscutible la necesidad de promover una formación que se concentre en el desarrollo humano integral de los estudiantes atendiendo no sólo las dimensiones cognitivas sino también las sociales y valorativas. Una formación que cultive el pensamiento crítico e independiente $\mathrm{y}$, a su vez, la capacidad de aprender a lo largo de toda la vida procurando para ello desarrollar capacidades que les permitan a los estudiantes avanzar en su propio aprendizaje y en su formación como personas comprometidas con la sociedad y la comunidad en la que se encuentran insertos.

Desde otro punto de vista, en lo que se refiere a las nociones menos relevantes, los estudiantes coinciden en que por debajo del resto de los conceptos se haya aquel que sugiere que la calidad consiste en conseguir la eficiencia, es decir, que ésta implicaría obtener los 


\section{CALIDAD DE LA EDUCACIÓN SUPERIOR: PERCEPCIONES DE LOS ESTUDIANTES \\ UNIVERSITARIOS ARGENTINOS \\ DOI: http://dx.doi.org/10.5007/1983-4535.2015v8n4p101}

mejores resultados con el menor uso de recursos. La mencionada definición, proveniente de la bibliografía de las ciencias empresariales, no parecería ser aceptada por los encuestados como válida al momento de analizar la calidad de la educación universitaria en Argentina.

En la mayoría de los casos, se advierte una gran disparidad entre el concepto con el cual se identifican los estudiantes y aquél con el cual consideran que se identifica su Facultad o Unidad Académica. Así por ejemplo, los estudiantes encuestados se identifican en un 84\% con el concepto de calidad que se refiere al desarrollo de las capacidades del estudiante para avanzar en su propia transformación, sin embargo consideran que su institución se identifica sólo en un 54\% con el mismo concepto. Asimismo los estudiantes adhieren en un $75 \%$ a que la calidad está altamente relacionada con la capacidad de formar estudiantes aptos para asumir un compromiso social, pero estiman que dicho concepto es importante o muy importante para su facultad simplemente en un 52\%.

Estas disparidades quedan en evidencia en el Gráfico 3 que se presenta a continuación.

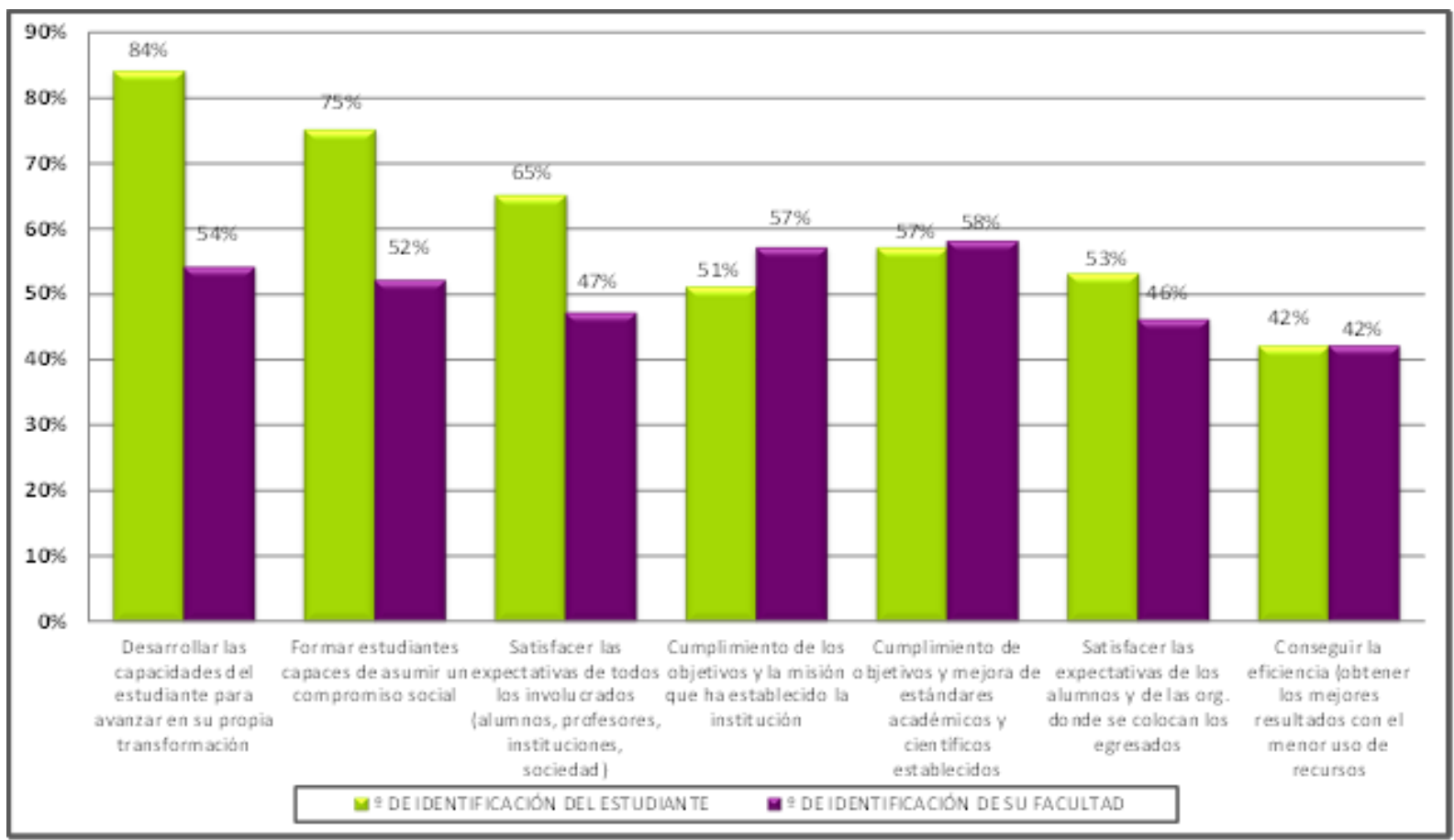

Gráfico 3 Grado de adhesión de los estudiantes a diferentes conceptos de calidad y el grado de identificación de su Facultad con los mismos (FRA)

Fuente: Elaboración propia

El caso donde se observa una mayor coincidencia entre la opinión de los estudiantes y la que atribuyen a la Facultad a la cual pertenecen es el de la calidad entendida como la eficiencia alcanzada obteniendo los mejores resultados con el menor uso de recursos. En 


\section{CALIDAD DE LA EDUCACIÓN SUPERIOR: PERCEPCIONES DE LOS ESTUDIANTES \\ UNIVERSITARIOS ARGENTINOS \\ DOI: http://dx.doi.org/10.5007/1983-4535.2015v8n4p101}

cuanto a esta concepción, los estudiantes consideran con FRA de $42 \%$ que esa noción es relevante, frecuencias que son equivalentes a las que perciben para sus Unidades Académicas.

\subsection{VALORACIÓN DE LA IMPORTANCIA RELATIVA DE FACTORES ASOCIADOS A LA CALIDAD DE LA ENSEÑANZA UNIVERSITÁRIA}

En este apartado se analizarán las opiniones de los alumnos sobre los factores que afectan a la calidad de la educación superior. Como complemento a las opiniones sobre la importancia de los factores que afectan a la calidad de la educación, se consultó a los encuestados sobre su percepción acerca de la presencia de dichos factores en su unidad académica.

En función de esta respuesta, se construyó una medida de "prioridad" de cada concepto, calculada mediante la diferencia entre el grado de importancia o incidencia y el nivel de presencia o desarrollo de los mismos. De este modo, aquellos factores valorados como importantes pero con un escaso desarrollo en las instituciones aparecerán como los de mayor prioridad. A continuación se exponen los resultados para los factores considerados como los prioritarios en las diferentes encuestas.

\begin{tabular}{|l|l|l|}
\hline IMPORT ANCIA & PRESENCIA & PRIORIDAD \\
\hline
\end{tabular}

\begin{tabular}{|l|c|c|c|}
\hline Motivación del profesor & $86 \%$ & $57 \%$ & $29 \%$ \\
\hline $\begin{array}{l}\text { Formación pedagógica actualizada del } \\
\text { profesor }\end{array}$ & $82 \%$ & $54 \%$ & $28 \%$ \\
\hline Capacidad de comunicación del profesor & $93 \%$ & $65 \%$ & $28 \%$ \\
\hline $\begin{array}{l}\text { Conocimiento del profesor sobre las } \\
\text { materias a su cargo }\end{array}$ & $96 \%$ & $87 \%$ & $9 \%$ \\
\hline $\begin{array}{l}\text { Participación del profesor en actividades de } \\
\text { extensión universitaria }\end{array}$ & $50 \%$ & $36 \%$ & $14 \%$ \\
\hline $\begin{array}{l}\text { Participación del profesor en actividades de } \\
\text { investigación }\end{array}$ & $58 \%$ & $47 \%$ & $11 \%$ \\
\hline $\begin{array}{l}\text { Promedio Factores Relacionados con el } \\
\text { Profesor }\end{array}$ & $78 \%$ & $58 \%$ & $20 \%$ \\
\hline
\end{tabular}




\section{CALIDAD DE LA EDUCACIÓN SUPERIOR: PERCEPCIONES DE LOS ESTUDIANTES UNIVERSITARIOS ARGENTINOS \\ DOI: http://dx.doi.org/10.5007/1983-4535.2015v8n4p101}

\begin{tabular}{|c|c|c|c|}
\hline $\begin{array}{l}\text { Existencia de servicios de apoyo adecuados } \\
\text { (biblioteca, gabinete de computación, } \\
\text { equipamiento, etc.) }\end{array}$ & $88 \%$ & $75 \%$ & $13 \%$ \\
\hline Rigor en la selección de los profesores & $80 \%$ & $44 \%$ & $36 \%$ \\
\hline $\begin{array}{l}\text { Capacitación brindada por la facultad o la } \\
\text { universidad a los profesores }\end{array}$ & $83 \%$ & $48 \%$ & $35 \%$ \\
\hline $\begin{array}{l}\text { Exigencia en el ingreso de los alumnos ala } \\
\text { facultad }\end{array}$ & $53 \%$ & $39 \%$ & $14 \%$ \\
\hline Coordinación entre profesores & $78 \%$ & $44 \%$ & $34 \%$ \\
\hline $\begin{array}{l}\text { Utilización de plat aformas tecnológicas de } \\
\text { apoyo a la docencia (campus virtual o }\end{array}$ & $61 \%$ & $60 \%$ & $1 \%$ \\
\hline $\begin{array}{l}\text { Agilidad para la gestión de trámites y } \\
\text { procesos administrativos }\end{array}$ & $75 \%$ & $41 \%$ & $34 \%$ \\
\hline $\begin{array}{l}\text { Coordinación de contenidos entre la } \\
\text { educación secundaria y la universitaria }\end{array}$ & $71 \%$ & $28 \%$ & $43 \%$ \\
\hline $\begin{array}{l}\text { Promedio Factores Relacionados con la } \\
\text { Institución Universitaria }\end{array}$ & $74 \%$ & $47 \%$ & $26 \%$ \\
\hline V ocación del alumno por la carrera elegida & $85 \%$ & $57 \%$ & $28 \%$ \\
\hline $\begin{array}{l}\text { Compromiso y actitud del alumno hacia el } \\
\text { aprendizaje a su ingreso }\end{array}$ & $89 \%$ & $48 \%$ & $41 \%$ \\
\hline $\begin{array}{l}\text { Participación activa del alumno en su } \\
\text { aprendizaje en el periodo universitario }\end{array}$ & $87 \%$ & $44 \%$ & $43 \%$ \\
\hline $\begin{array}{l}\text { Conocimientos generales del alumno a su } \\
\text { ingreso en la universidad }\end{array}$ & $67 \%$ & $23 \%$ & $44 \%$ \\
\hline $\begin{array}{l}\text { Existencia de mecanismos de evaluación de } \\
\text { los profesores (evaluaciones por parte de los } \\
\text { alumnos, por parte de la facultad o por parte } \\
\text { de otras instituciones) }\end{array}$ & $75 \%$ & $40 \%$ & $35 \%$ \\
\hline $\begin{array}{l}\text { Promedio Factores Relacionados con los } \\
\text { estudiantes }\end{array}$ & $81 \%$ & $42 \%$ & $38 \%$ \\
\hline
\end{tabular}

Cuadro 3 Percepciones de los estudiantes sobre factores prioritarios que influyen en la calidad de la enseñanza universitaria

Fuente: Elaboración propia em base a datos obtenidos em la investigación CESPEA

Con respecto a la prioridad de los factores vinculados a los profesores, en el Cuadro 3 se evidencia que los alumnos consideran que estos factores no tendrían una alta prioridad en su facultad, ya que las mismas no superan en ningún caso el 30\%. En otras palabras, si bien se observa que para los estudiantes esta categoría de factores es muy importante para la calidad, dado que el promedio de FRA es $78 \%$, dichos actores también consideraron que tales 
elementos son los que poseen un mayor nivel de desarrollo o presencia en sus unidades académicas.

Los resultados referidos al segundo grupo propuesto, el vinculado a los factores prioritarios asociados a la Institución Universitaria, tampoco tienen una alta prioridad si se analiza en conjunto. A pesar de ello, y realizando un análisis individual de cada factor, se observa que le dan una significativa importancia al factor "coordinación de contenidos entre la educación secundaria y la universitaria" pero consideran que su desarrollo es escaso en sus respectivas Facultades, por lo que resulta ser un área prioritaria dentro de los factores vinculados a la Institución. Lo mismo pareciera suceder, en menor escala, con los factores "capacitación brindada por la Facultad o la universidad a los profesores" y "rigor en la selección de los profesores".

Por último, se presentan las opiniones de los encuestados respecto al grado de prioridad de los factores asociados a los estudiantes. En el mismo surge que consideran que todos los factores de la categoría aludida, deben ser considerados como prioritarios en sus instituciones (con niveles superiores al 35\%), excepto la "vocación del alumno por la carrera elegida" dado que si bien es considerado, por estos como muy importante para la calidad de la educación superior, también estiman que la misma se encuentra relativamente presente en las instituciones a las que pertenecen.

Así entonces, podría concluirse que, en su mayoría, los encuestados perciben que los elementos que integran este grupo resultan fundamentales y determinantes para la calidad educativa pero, sin embargo, en la actualidad, su nivel de presencia es considerablemente menor al esperado, por lo cual las respectivas Unidades Académicas deberían procurar una intervención más activa en este sentido.

\section{CONCLUSIONES}

A lo largo de este trabajo se han presentado los resultados de un proyecto de investigación sobre la calidad de la enseñanza universitaria realizado en Argentina (Proyecto CESPÈA). Específicamente se han abordado las percepciones de los estudiantes de la Universidad Nacional de La Plata.

No cabe duda que, actualmente, nos encontramos inmersos en una sociedad del conocimiento y en este marco, las universidades asumen el papel protagónico que las industrias supieron ocupar en la era de la sociedad industrial. El trabajador de esta era, 
"trabajador del conocimiento" como lo denomina Peter Drucker (1993), recurre a su intelecto más que a su fuerza, por lo cual, el debate sobre la problemática de la educación superior en general y de la calidad educativa universitaria en particular, no puede ser dejado de lado en la agenda de ningún país.

Ahora bien, más allá de que existe consenso en este sentido, no parecería haber parámetros cuantitativos para medir dicha calidad de la enseñanza y, más importante aún, parecería existir dificultades para arribar a un acuerdo generalizado sobre la conceptualización de este término. Frente a esto, el grupo de investigación de la Red ECUALE, ha considerado que las opiniones de los diferentes estamentos involucrados en el proceso posibilitan una aproximación útil para la medición y evaluación de la calidad de la educación superior.

En este sentido, parece justo y razonable atribuir a los estudiantes universitarios un grado de conocimiento y una capacidad de análisis tales que los sitúan en condiciones idóneas para interpretar con acierto cuáles son las variables que actúan como factores de calidad en el proceso de enseñanza y aprendizaje. De todas maneras y no obstante ello, las respuestas que se han recogido deben ser entendidas como opiniones particulares pero cualificadas sobre la causalidad que opera detrás de dicho proceso y sus resultados.

Fundamentalmente, el valor agregado que pretende aportar este artículo radica en la posibilidad de ofrecer información empírica sobre los aspectos centrales que giran en torno a la conceptualización de la calidad educativa y sobre los factores o elementos que la determinan.

En cuanto a los resultados obtenidos, el análisis efectuado ha revelado en primer término que, una alta proporción de los estudiantes encuestados, asocia el concepto de calidad con la posibilidad de generar un espíritu crítico en los estudiantes, con la posibilidad de dotarlos de capacidades para el aprendizaje autónomo y con la capacidad para hacerlos asumir un fuerte compromiso social.

En cuanto a los principales factores que incidirían en la calidad universitaria, estos han manifestado que consideran importantes tanto aquellos que hacen al profesor como a los asociados a los propios estudiantes. Sin embargo, es en este último grupo de factores donde consideran que, dada su escaza presencia en las instituciones, debería comenzar a trabajarse e intervenirse con mayor urgencia y prioridad.

En síntesis se puede decir que, tanto desde el punto de vista conceptual, como desde el de los factores, lo estudiantes parecerían estar demandando que el proceso educativo 
comience a tener un mayor registro de sus condiciones y características y la incidencia que las mismas poseen para la calidad educativa. Cuestiones que se encontrarían en consonancia con las teorías más modernas en educación las cuales ponen el foco de atención en los propios estudiantes y comienzan a dejar de lado la mirada únicamente centrada en el profesor, que conciben a éste como el receptáculo de todo el conocimiento y único capaz de poder generarlo y transmitirlo.

\section{BIBLIOGRAFÍA}

Barrenetxea, M. (2005): Modelos de calidad y evaluación de la educación superior en la Unión Europea, Tesis Doctoral. Universidad del País Vasco. Bilbao.

Drucker, Peter F. (1993) La Sociedad Poscapitalista. Editorial Sudamericana, Buenos Aires.

Gento Palacios, S. (1996) "Liderazgo Pedagógico para la Calidad Educativa” en Pérez Ferra, M y Ruiz Carrascosa, J. (Coords.). Factores que Favorecen la Calidad Educativa. Jaén: Universidad de Jaén. (pp. 201-225).

Harvey, L. y Green, D. (1993). "Defining quality". En Assessment and Evaluation in Higher Education, 18 (1). P: $9-34$.

López Armengol, M. A. y Colombo, M. P. (coords.) (2011). "Hacia una Educación Superior de Calidad (Volumen II). Una mirada de quienes gestionan las universidades en Argentina, España y México. (Proyecto CESPUALE)". Editorial EDULP (Editorial de la Universidad Nacional de La Plata). La Plata, Argentina.

Martínez, M. C; y Romero, M. A. (1999): “Calidad de la educación e integración de las nuevas tecnologías”, Comunicar, 13: 183-187

Mora Ruiz, J.G. (1991) "Calidad y Rendimiento en las Instituciones Universitarias". Consejo de Universidades. Madrid

Pérez Ferra, M y Ruiz Carrascosa, J. (Coords.) (1996). "Factores que Favorecen la Calidad Educativa”. Jaén: Universidad de Jaén

Torres González, J.A. (1996) "La Formación del Profesorado como Factor Favorecedor de la Calidad Educativa" en Pérez Ferra, M y Ruiz Carrascosa, J. (Coords.). Factores que Favorecen la Calidad Educativa. Jaén: Universidad de Jaén (pp. 69-132). 